\title{
Effect of push-up exercises on hand grip strength in diplegic children.
}

\author{
Ramy H Hegazy ${ }^{*}$, Emam H El-Negamy ${ }^{1}$,Hebatallah M Kamal ${ }^{1}$, Amina S Hendawy ${ }^{2}$ \\ ${ }^{1}$ Faculty of Physical Therapy, Department of Physical Therapy for Pediatrics, Cairo University, Egypt \\ ${ }^{2}$ Faculty of Medicine, Department of Pediatrics, Cairo University, Egypt
}

\begin{abstract}
Background: Diplegia is a spastic form of cerebral palsy with lower limbs more affected than upper limbs. The hand is effector organ of upper extremity for support, manipulation and prehension.

Purpose: The purpose of this study is to determinethe effect of push-up exercises on hand grip strength in spastic diplegic cerebral palsied children.

Subjects and methods: Sixty spastic diplegic children with age ranged from 4 to 7 years from both sexes participated in this study. They were chosen from physical therapy department, Mubarak Central Hospital, Giza Governorate, Egypt. Subjects were divided randomly into three groups A, B and C, of equal number [twenty patients each]. Each patient was evaluated pre and post-treatment individually by using Peabody Developmental Motor Scale (PDMS-2) to determine standard scores of grasping subtest and age equivalent for fine motor activities and Baseline pneumatic squeeze handheld dynamometer to detect maximum hand grip strength. Control group (A) received traditional physiotherapy program for spastic diplegia and the two study groups $(B$ and $C)$ received the same traditional physiotherapy program in addition to push-up exercises on stable surface for group B in form of wide base push-up exercises, while group $C$ in form of narrow base push-up exercises. The treatment protocol was conducted for three successive months and six times per week.

Results: The results of this study revealed significant improvement in hand grip strength, standard scores of grasping and age equivalent in the three groups equally.

Conclusion: Conventional physiotherapy program is effective as push-up exercises in improving hand function in spastic diplegic children. Push-up exercises only do not add benefits on hand grip strength.
\end{abstract}

Keywords: Spastic diplegia, Hand grip strength, Push-up exercises, Peabody Developmental Motor Scale (PDMS-2), Baseline pneumatic squeeze handheld dynamometer.

Accepted on March 14, 2019

\section{Introduction}

Spastic diplegia is the common term applied to variation of spastic quadriparesis in which the lower limbs are more affected than upper limbs. Those with spastic diplegia have greater lower extremity involvement than upper extremity and eventually become ambulatory and it account for approximately $50 \%$ of total cerebral palsy population [1]. Spastic diplegia is the most common pattern of motor impairment in patients with cerebral palsy due to several deficits including poor muscle control, weakness, impaired balance, hypertonicity and spasticity [2]. Upper limbs play an important role in everyone's daily life. Several sensorimotor parameters, including hand grip strength, are necessary for their optimal performance [3]. Almost all the children with diplegia were bilaterally impaired in fine finger dexterity, with slightly more than half of them showing bilateral impairments in gross manual dexterity and grip strength [4]. Proper handgrip strength is essential for carrying precise hand functions such as gripping and pulling. It is considered a crucial factor in maximizing performance and control of many daily activities and sporting [5]. Hand Grip Strength [HGS] is the maximal power of forceful voluntary flexion of all fingers under normal biokinetic conditions [6]. Hand Grip Strength [HGS] is one of the best indicators of the overall strength of the upper limb and it is evaluated as a component of hand function according to American Society of Hand Therapists (ASHT) [7]. The use of Hand Grip Strength is a single, simple and inexpensive method for assessing general muscle strength and function [8]. The Baseline pneumatic squeeze handheld dynamometer is used to assess hand grip strength and is easier to use and much lighter and hence more applicable in the primary care setting later for routine use [9]. Fine motor skills refer to finger dissociation and grading of muscle activity coordinated with fixation at wrist, elbow, and shoulder in order to ensure a mature stable functional pencil grasp [10]. The Peabody Developmental Motor Scale (PDMS-2) is a valid and reliable tool for assessment of developmental activities in children from birth to 72 months old [11]. The tasks constructing PDMS-2 included various performance 
components related to grasping [neuromuscular and neurodevelopmental status, perception, motor performance, visual motor integration, bilateral integration and sensory processing]. Items of PDMS-2 identify all the aspects of performance components within the context of play-based assessment [12]. Push-up exercises are very popular in upper body strengthening programs. They are closed kinetic chain exercises, for which pectoralis major and triceps brachii are the principle acting muscles [13]. The wide base push-up exercises isolated pectoralis major muscle [14] and the narrow base push-up exercises isolated triceps brachii [15]. Hand weight bearing improved co-contraction at the scapula-humeral area, the elbow and the wrist [16].

\section{Materials and Methods}

Sixty children from both sexes with mild spastic diplegia were selected in this study. We considered age of selected children from 4 to 7 years because this preschool period is very important for formation of personality and children build the motor abilities and later, they only learn a variety of modifications and adjustments to various situations in life. This study was done in physical therapy department, Mubarak Central Hospital, Giza Governorate, They were able to sit without support, they could achieve minimal active grasp and they were able to understand and follow verbal commands. Those had significant tightness or deformity of upper limbs and spine were excluded. Children were divided randomly to the three groups (20 children each), Control group A received only traditional physiotherapy program for spastic diplegia, Study group B received the same program plus push-up exercises on mate with wide base hand position (hands 5 inch laterally from the shoulder width position, where the middle finger at the end point of a plumb line hung from the edge of their deltoid) and study group $\mathrm{C}$ received the same program plus push-up exercises on mate with narrow base hand position (hands together under the center of sternum, forming diamond shape between their thumbs and index fingers). All subjects were evaluated before and after 3 successive months of the selected program by Peabody Developmental Motor Scale (PDMS-2) and Baseline Pneumatic Squeeze Handheld Dynamometer. Baseline Pneumatic Squeeze Handheld Dynamometer (PDMS-2) to assess fine motor skills. Grasping subtest of PDMS-2 was used in this study, measures the child ability to use his hands and fingers. It consists of 26 items with total raw scores 52. Each child was asked to sit on chair in front of table and follow subtests according to entry, basal and ceiling points to shorten time of test. Finally, examiner collected all scores and represented them to standard scores of grasping subtest and age equivalent scores in months. Maximum hand grip strength was evaluated by Baseline Pneumatic Squeeze Handheld Dynamometer (model 12-0290 USA) as shown in Figure 1, it is soft rubber bulb, $13 \mathrm{~cm}$ in circumference and easy to grip and squeeze. It is calibrated in pounds per square inch (PSI) indicating the pressure is relative to atmospheric pressure [9]. Child is asked to sit on the chair with a straight back, without armrest and with the feet flat on the floor, shoulder adducted and neutrally rotated, elbow flexed at 90 , forearm and wrist in neutral position as recommended by American Society of Hand Therapy (ASHT) [17]. The examiner instructed the child to squeeze the handle of dynamometer with maximum force and sustain this for 3-5 seconds per each trial and no break between trials ( 3 trials for each hand) with a rest of 15-20 seconds in between measurements of both hands [18]. Three successive measurements were taken for each hand [dominant hand then non-dominant hand], the maximum of the three grips of each hand recorded, as the dominant hand has a $10 \%$ stronger grip than the non-dominant hand [19]. Traditional physiotherapy program included stretching of tight muscles, exercises to encourage standing and self-support, balance and vestibular exercises and gait training. Traditional physiotherapy program was received 6 times per week (2 hours daily). Push-up exercises was used in groups $\mathrm{B}$ and $\mathrm{C}$ only, for 6 sessions per week, 8 minutes per session, 5 sets per session, and 10 repetitions per each set with 30 seconds rest in between sets. Child lifted the body from pelvis up (fixed point) to prevent any compensation movements until arms fully extended then slowly return his body to the floor, shoulders and trunk moved as one unit.

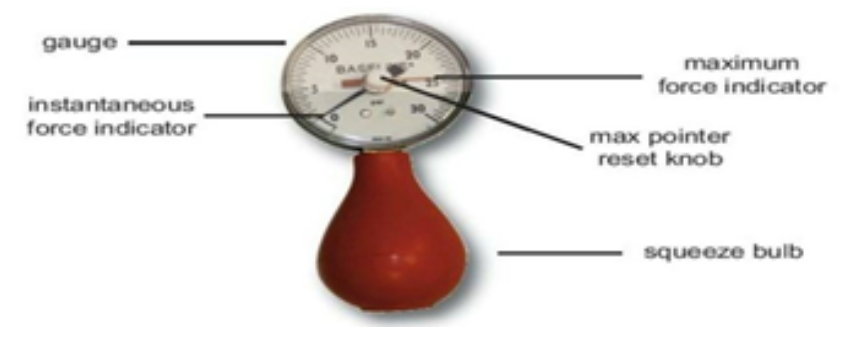

Figure 1. Baseline pneumatic squeeze handheld dynamometer.

\section{Statistical methods}

ANOVA was carried out to compare between three groups in selected variables (Hand grip strength, Standard scores of grasping and Age equivalent). Pearson Correlation Coefficient was conducted to determine the correlation between hand grip strength, standard score of grasping and age equivalent. The level of significance for all statistical tests was set at $\mathrm{p}<0.05$. All statistical tests were performed through the statistical package for social studies (SPSS) version 19 for windows. (IBM SPSS, Chicago, IL, USA).

\section{Results}

There was no significant difference between three groups in mean values of pre-treatment hand grip strength bilaterally (Figure 2). There was no significant difference between three groups in mean values of post treatment hand grip strength bilaterally (Figure 3). There was no significant difference between three groups in mean values of pre-treatment standard scores of grasping bilaterally (Figure 4). 


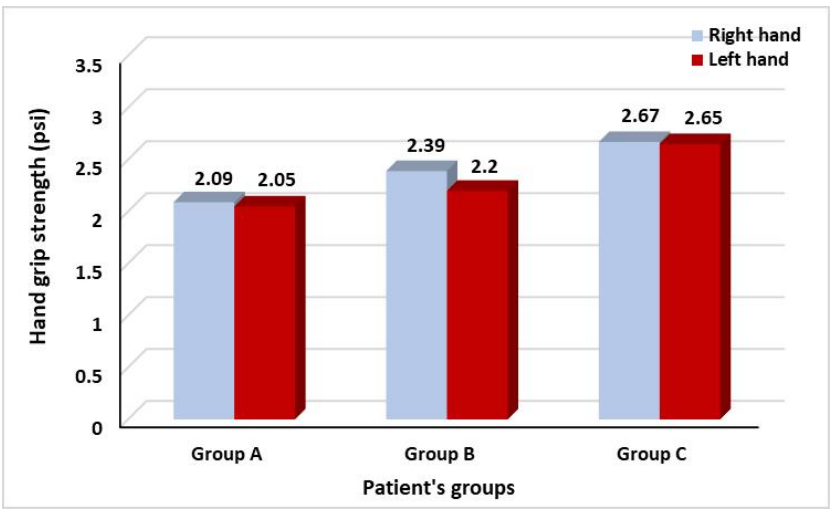

Figure 2. Pre-treatment mean values of hand grip strength.

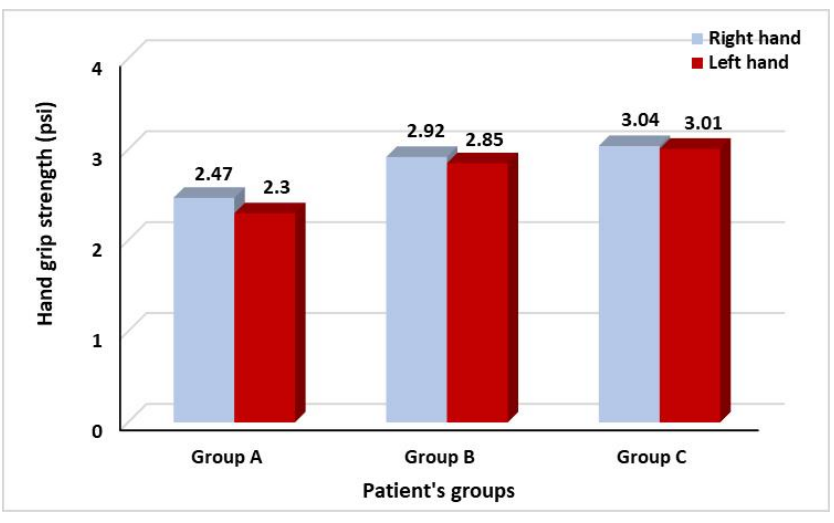

Figure 3. Post-treatment mean values of hand grip strength.

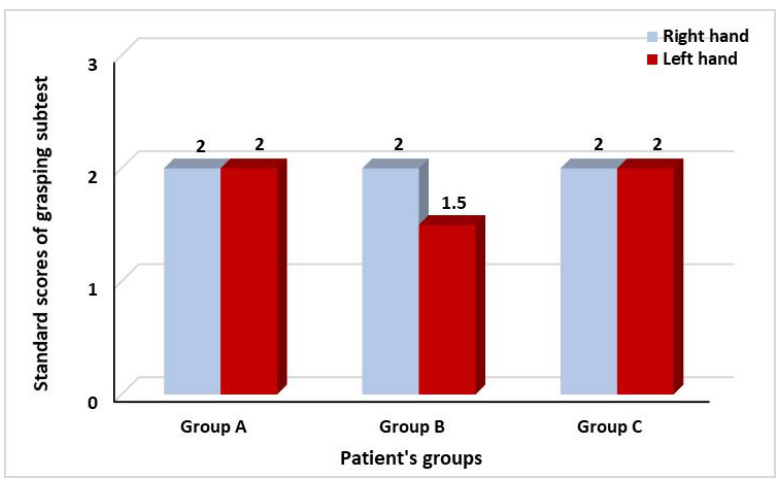

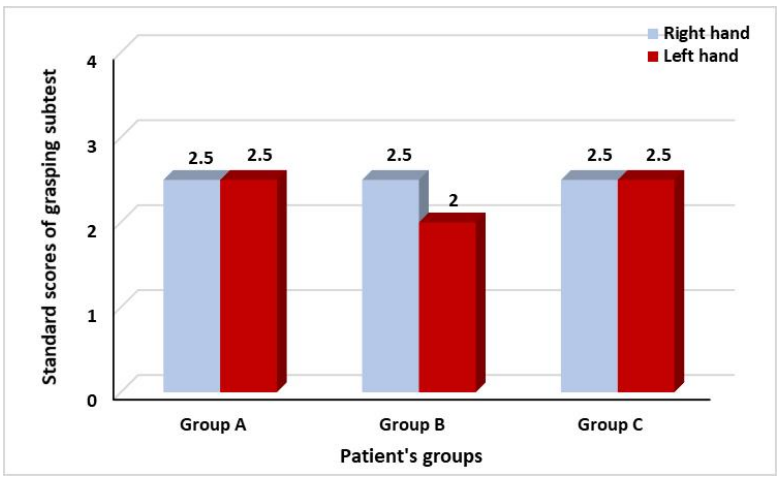

Figure 5. Post-treatment mean values of standard scores.

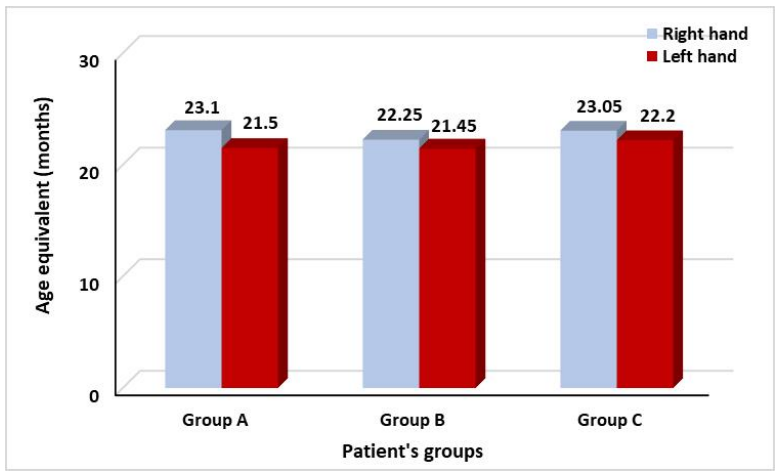

Figure 6. Pre-treatment mean values of age equivalent.

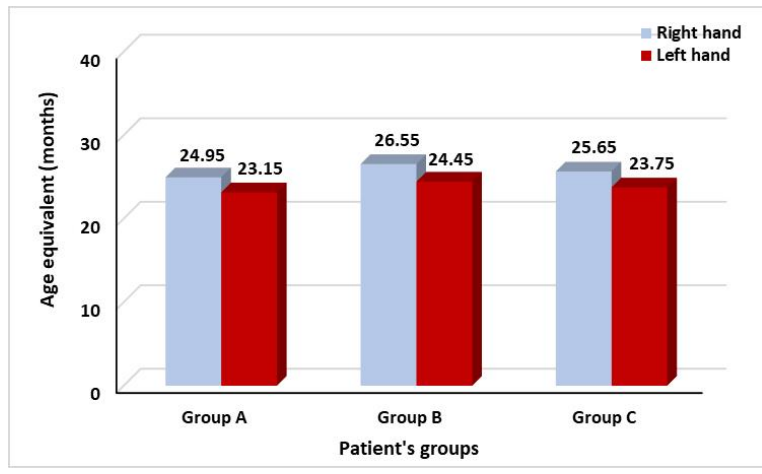

Figure 7. Post-treatment mean values of age equivalent.

Figure 4. Pre-treatment mean values of standard scores.

Table 1. Correlation between right hand grip strength and other variables.

\begin{tabular}{|c|c|c|c|c|}
\hline Right hand & Grasping variables & $r$ value & $p$ value & Significance \\
\hline \multirow{2}{*}{ Hand grip strength (psi) } & Standard scores of grasping & 0.59 & 0.0001 & $S$ \\
\hline & Age equivalent (months) & 0.48 & 0.0001 & $\mathrm{~S}$ \\
\hline
\end{tabular}

Table 2. Correlation between left hand grip strength and other variables.

\begin{tabular}{llcc}
\hline Left hand & Grasping variables & r value & S value \\
\hline Hand grip strength $(\mathrm{psi})$ & Standard scores of grasping & 0.39 & 0.002 \\
\hline
\end{tabular}


There was no significant difference between three groups in mean values of post treatment standard scores of grasping bilaterally (Figure 5). There was no significant difference between three groups in mean values of pre-treatment age equivalent bilaterally (Figure 6). There was no significant difference between three groups in mean values of post treatment age equivalent bilaterally (Figure 7). There was moderate positive significant correlation between hand grip strength and standard scores of grasping in right hand and left hand respectively $(p=0.0001, p=0.002)$. There was moderate positive significant correlation between hand grip strength and age equivalent of grasping in right hand and left hand respectively $(\mathrm{p}=0.0001, \mathrm{p}=0001)$. As illustrated in Tables 1 and 2.

\section{Discussion}

Children with spastic diplegia demonstrate asymmetric motor abilities, with one lower limb often more affected than the other. The side-effect differences in motor control may not be visibly manifest during weight bearing, as the reflex activation of extensor musculature is dynamic in nature. Therefore, functional asymmetry may be difficult to recognize in qualitative, subjective assessment. Also, co-contraction of antagonistic muscle pairs may be the limiting factor in the range of voluntary joint movement [20]. Performing push-up exercises on fixed pelvis as a kinetic chain rehabilitation exercises is to perform movement patterns without compensations through altering the dominant plane of motion, posture, resistance and tactile or verbal feedback [21]. Pretreatment results showed non-significant differences between the three groups $(\mathrm{A}, \mathrm{B}, \mathrm{C})$ in standard scores of grasping skills, age equivalent in months and hand grip strength bilaterally which confirm the validity of the used patient sample. The non-significant pre-treatment results of HGS may be because that pneumatic instrument relay on compression of an air-filled compartment and thereby measure grip pressure than grip strength. The limitation is that varying the surface area over which pressure is applied leads to variable results [22]. The non-significant results of pre-treatment standard scores of grasping skills and age equivalent due to developmental delay of spastic diplegia which characterized by poor timing and delayed muscle onset latencies [23]. Children with spastic diplegia have only a moderate delay in gross motor domain and mild delay in other domains. In addition, motor functions including gross motor and fine motor functions are significantly related to self-help ability which was affected [24]. The non-significant difference in post treatment mean values of HGS between three groups may be because that all subjects of spastic diplegia were limited in their activity due to more protection from parents or neglection toward affection of upper limbs. Thus, use of both hands is limited with traditional play with cup and spoon or big ball [25]. Physical play is a major occupation in children at this age. Pre-school children are extremely active and always engage in rough and tumble play. They continue to delight in movement experiences that provide strong sensory input [26]. This revealed that no benefit of push-up exercises on hand grip strength in diplegic children. Play activities allow children to use their creativity while developing their imagination, dexterity and physical, cognitive and emotional strength. Play is important for healthy brain development [27]. The post treatment mean values of age equivalent and standard scores of the three groups (A,B and C) revealed non-significant difference. This may be attributed to a one-dimensional awareness of the environment developed by preschool children. They use symbols and internal representations to think, but thinking is illogical and unsystematic according to piaget's stage theory of cognitive development, but school aged 7-11 years children accumulate physical experience and accommodation increased. That means school children begins to think abstractly and conceptualize, creating logical structures that explain his or her physical experience [28]. Positive correlation between HGS and standard scores of grasping and age equivalent may be due to adaptation which is a physiologic change in perception of stimulus, occurs in response to constant stimulation. Adaptation requires one to adjust environmental conditions and intellectualize the adjustments through the complementary processes of accommodation and assimilation [29].

\section{Conclusion}

Push-up exercises do not add benefits over conventional therapy on improvement of hand grip strength in diplegic children in preschool age (4-7 years). Play activities and practice play important role in improving fine motor skills.

\section{References}

1. Damiano DL, Martellotta TL, Sullivan DJ, Granata KP, Abel MF. Muscle force production and functional performance in spastic cerebral palsy: Relationship of cocontraction. Arch Phys Med Rehabil 2000; 81:895-900.

2. Baddar A, Granata K, Damiano DL, Carmines DV, Blanco JS, Abel MF. Ankle and knee coupling in patients with spastic diplegia, Effects of gastrocnemius soleus lengthening. The journal of bone and joint surgery 2002; 84:251-259.

3. Wallen M, Stewart K. upper limb function in everyday life of children with cerebral palsy: description and review of parent report measures. Disabil Rehabil 2015; 37:1353-1361.

4. Arnould C, Penta M, Thonnard J. Hand impairments and their relationship with manual ability in children with cerebral palsy. Neuro med 2015; 39:708-714.

5. Al-Asadi JN. Handgrip strength in medical students: Correlation with body mass index and hand dimensions. Asian J Med Sci 2018; 9:21-26. 
6. Sengupta DS, Maity P, Pal P, and Dhara PC. Effect of body posture on hand grip strength in adult Bengalee population. J Exer Sci Physiother 2011; 7:79-88.

7. Martins JC, Teixeira-Salmela LF, Araújo L, Aguiar LT, Lara EM, Moura JB, de Morais F, Coelho CD. Reliability and validity of the modified sphygmomanometer test for assessment of strength of upper limb muscles after stroke. J Rehabil Med 2015; 47:697-705.

8. Aadahl M, Beyer N, Linneberg A, Thuesen BH, Jørgensen T. Grip strength and lower limb extension power in 19 72-year-old Danish men and women: the Health2006 study. BMJ open 2011; 1:e000192.

9. Wahba H, Abdul-Rahman S, Mortagy A. Handgrip strength and falls in community-dwelling Egyptian seniors. Adv Aging Res 2013; 2:109.

10. Schwellnus H, Carnahan H, Kushki A, Polatajko H, Missiuna C, Chau T. Writing forces associated with four pencil grasp patterns in grade 4 children. American $\mathrm{J}$ Occupational Ther 2013; 67: 218-227.

11. Folio MR, Fewell RR. Peabody Developmental Motor Scales: Examininer's Manual 2nd ed Austin, Tex: PROED. Motor profile of Portuguese preschool children on the Peabody Developmental Motor Scales-2: A cross-cultural study 2013.

12. Tomchek SD, Schneck CM. Evaluation of Handwriting. In Henderson $\mathrm{A}$ and Pehoski C: Hand Function in the Child: Foundations for Remediation Mosby 2006.

13. Youdas JW, Budach BD, Ellerbusch JV, Stucky CM, Wait KR, Hollman JH. Comparison of muscle-activation patterns during the conventional push-up and perfect. pushup ${ }^{\mathrm{TM}}$ exercises. J Strength Cond Res 2010; 24:3352-3362.

14. Hendel AP. Living better with the push-up. 2004.

15. Weede T, Kraemer WJ. Muscle and fitness blueprint for arms. Muscle Fitness 2002.

16. Azzam AM. Effect of hand function training on improvement of hand grip strength in hemiplegic cerebral palsy in children. J Nov Physiother 2012; 2:116.

17. El-Sais WM, Mohammad WS. Influence of different testing postures on hand grip strength. Eur Sci J 2014; 30:10.

18. Tsang RC. Reference values for 6-minute walk test and hand-grip strength in healthy Hong Kong Chinese adults. Hong Kong Physiother J 2005; 23:6-12.

19. Roy JS, MacDermid JC, Orton B, Tran T, Faber KJ, Drosdowech D, Athwal GS. The concurrent validity of a hand-held versus a stationary dynamometer in testing isometric shoulder strength. J Hand Ther. 2009; 22:320-326.

20. Cowan MM, Stilling DS, Naumann S, Colborne GR. Quantification of antagonist muscle co-activation in children with spastic diplegia. Clinical Anatomy 1998; 11:314-319.

21. Mcmullen J, Timothy L. A kinetic chain Approach for shoulder Rehabilitation; Athletic training J 2000; 35:329-337.

22. Fess EE. Documentation: essential elements of an upper extremity assessment battery. Rehabilitation of the hand: surgery and therapy $1995 ; 1: 185-214$.

23. Palisano RJ. Movement sciences: Transfer of knowledge into pediatric therapy practice. Routledge 2004; 24:23.

24. Lee YC, Wu CY, Liaw MY, Lin KC, Tu YW, Chen CL, Chen CY, Liu WY. Developmental profiles of preschool children with spastic diplegic and quadriplegic cerebral palsy. Kaohsiung J Med Sci 2010; 26:341-349.

25. Roberts HC, Denison HJ, Martin HJ, Patel HP, Syddall H, Cooper C, Sayer AA. A review of grip strength in clinical and epidemiological studies: towards a standard approach. Age Ageing 2011; 40:423-429.

26. Case-Smith J, O'Brien JC. Occupational therapy for children. Maryland Heights MO: Mosby 2010.

27. Ginsburg R. The importance of play in prompting healthy child development and maintaining strong parent-child bonds. American Acad pediatr 2007; 119-182.

28. Bala G. Structural differences in motor skills for boys and girls in preschool age. In Serbian Pedagoškastvarnost 2002; 48:744-752.

29. Probst R, Grevers G, Iro H. Basic otorhinolaryngology; a step by step learning guide. 2nd ed thieme 2004; 173-175.

\section{*Correspondence to:}

\section{Ramy H Hegazy}

Department of Physical therapy for Pediatrics

Cairo University

Egypt

E-mail: ramyhegazy93@gmail.com 